\title{
Simplified damage plasticity model for concrete
}

\begin{abstract}
The past several years have witnessed an increase in research on the nonlinear analysis of the structures made from reinforced concrete. Several mathematical models were created to analyze the behavior of concrete and the reinforcements. Factors including inelasticity, time dependence, cracking and the interactive effects between reinforcement and concrete were considered. The crushing of the concrete in compression and the cracking of the concrete in tension are the two common failure modes of concrete. Material models were introduced for analyzing the behavior of unconfined concrete, and a possible constitutive model was the concrete damage plasticity (CDP) model. Due to the complexity of the CDP theory, the procedure was simplified and a simplified concrete damage plasticity (SCDP) model was developed in this paper. The SCDP model was further characterized in tabular forms to simulate the behavior of unconfined concrete. The parameters of the concrete damage plasticity model, including a damage parameter, strain hardening/softening rules, and certain other elements, were presented through the tables shown in the paper for concrete grades B20, B30, B40 and B50. All the aspects were discussed in relation to the effective application of a finite element method in the analysis. Finally, a simply supported prestressed beam was analyzed with respect to four different concrete grades through the finite element program. The results showed that the proposed model had good correlation with prior arts and empirical formulations.
\end{abstract}

Keyword: Concrete damage plasticity; Concrete failure; Unconfined concrete; Fnite element analysis; Crack; Crush 